\title{
The Risk of Specific Congenital Anomalies in Relation to Newer Antiepileptic Drugs: A Literature Review
}

\author{
Josta de Jong $^{1}$ - Ester Garne ${ }^{2}$ Lolkje T. W. de Jong-van den Berg ${ }^{1}$. \\ Hao Wang ${ }^{1}$
}

Published online: 24 May 2016

(c) The Author(s) 2016. This article is published with open access at Springerlink.com

\begin{abstract}
Background More information is needed about possible associations between the newer anti-epileptic drugs (AEDs) in the first trimester of pregnancy and specific congenital anomalies of the fetus.

Objectives We performed a literature review to find signals for potential associations between newer AEDs (lamotrigine, topiramate, levetiracetam, gabapentin, oxcarbazepine, eslicarbazepine, felbamate, lacosamide, pregabalin, retigabine, rufinamide, stiripentol, tiagabine, vigabatrin, and zonisamide) and specific congenital anomalies.

Methods We searched PubMed and EMBASE to find observational studies with pregnancies exposed to newer AEDs and detailed information on congenital anomalies. The congenital anomalies in the studies were classified according to the congenital anomaly subgroups of European Surveillance of Congenital Anomalies (EUROCAT). We compared the prevalence of specific congenital anomalies in fetuses exposed to individual AEDs in the combined studies with that of the general population in a reference database. A significantly higher prevalence based on three or more fetuses with anomalies was considered a signal.

Results Topiramate showed a higher rate of congenital anomalies than the other newer AEDs. Four signals were
\end{abstract}

Josta de Jong

josta.de.jong@rug.nl

1 Department of Pharmacoepidemiology and Pharmacoeconomics, University of Groningen, A. Deusinglaan 1, 9713AV Groningen, The Netherlands

2 Paediatric Department, Hospital Lillebaelt, Kolding, Denmark found. The signals for associations between topiramate and cleft lip with/without cleft palate and hypospadias were considered strong. Associations between lamotrigine and anencephaly and transposition of great vessels were found within one study and were not supported by other studies. No signals were found for the other newer AEDs, or the information was too limited to provide such a signal.

Conclusion In terms of associations between monotherapy with a newer AED in the first trimester of pregnancy and a specific congenital anomaly, the signals for topiramate and cleft lip with/without cleft palate and hypospadias should be investigated further.

\section{Key Points}

Information was found on specific congenital anomalies in fetuses exposed to lamotrigine, topiramate, levetiracetam, gabapentin, and oxcarbazepine monotherapy in the first trimester.

The possible association between cleft lip and hypospadias and the use of topiramate in pregnancy should be investigated further.

\section{Introduction}

Users of anti-epileptic drugs (AEDs) regularly include women of childbearing age. During pregnancy, the treatment of epilepsy often needs to be continued, and-if possible-a pregnant woman with epilepsy should be free of seizures, because a seizure could harm both mother and fetus [1]. On the other hand, harm to the fetus from the use 
of medication in pregnancy should also be minimized. A balance must be found between benefits and risks for mother and child through agreement between the pregnant woman and the physician [2, 3]. Objective scientific information on the risks of AEDs to the fetus is essential for these discussions and decisions.

AEDs are classified in two groups: older AEDs and newer AEDs, the latter being introduced during the last two decades. Since the late 1990s, the use of newer AEDs has increased, especially for indications other than epilepsy such as neuropathic pain, mood disorders, migraine, and depression [4-6]. Lamotrigine, topiramate, gabapentin, and pregabalin are the most commonly used newer AEDs [4]. Women in particular seem to use more newer AEDs than do men, probably in an effort to avoid the use of valproate during childbearing years [7, 8]. About $0.5 \%$ of pregnant women in Europe use AEDs, most often carbamazepine, valproic acid, or lamotrigine; the most frequently used AEDs differ between countries [9].

More information is needed on the risk of congenital anomalies in exposed pregnancies for the newer AEDs. These newer AEDs might have an increased risk for specific congenital malformations. We performed an extensive literature search to find signals of higher risks of specific congenital anomalies in relation to the use of newer AEDs in pregnancy.

\section{Methods}

\subsection{Search Strategy}

The newer AEDs included in this review were lamotrigine, topiramate, levetiracetam, gabapentin, oxcarbazepine, eslicarbazepine, felbamate, lacosamide, pregabalin, retigabine, rufinamide, stiripentol, tiagabine, vigabatrin, and zonisamide.

We searched PubMed for original articles using the following search strategy:

1. congenital abnormalities [medical subject heading; $\mathrm{MeSH}$ ] OR pregnancy complications/drug therapy OR pregnancy complications/drug effects OR pregnancy outcome $[\mathrm{MeSH}]$

AND

2. (felbamate OR gabapentin OR lacosamide OR lamotrigine OR levetiracetam OR pregabalin OR topiramate OR vigabatrin OR eslicarbazepine OR oxcarbazepine OR rufinamide OR stiripentol OR zonisamide OR tiagabine OR retigabine OR pheneturide) NOT (models, animal $[\mathrm{MeSH}]$ OR animal experimentation $[\mathrm{MeSH}])$.
We searched EMBASE for all newer AEDs separately using the following search strategy:

'lamotrigine (or other drug) AND congenital malformation AND pregnancy NOT review'.

The search was conducted on 12 November 2014.

\subsection{Selection}

The articles were selected using the following inclusion criteria:

- original randomized controlled trials or observational studies.

- exposure to a newer AED as monotherapy in the first trimester ( $\leq 12$ weeks of gestation).

- information on congenital anomalies.

- the most recent update of studies based on the same long-term databases or pregnancy registries.

- enrolment of the pregnant women before the outcome of the pregnancy was known in the cohort and observational studies.

The articles from PubMed were selected based on the title and abstract. They were classified into two groups (studies including one AED and studies including more than one AED) and were read carefully, including the appendices. The articles were selected based on the inclusion criteria and categorised per AED; thus, the articles with more than one AED were categorised more than once. Additional articles found in EMBASE or the references that met the inclusion criteria were added (Fig. 1).

\subsection{Data Extraction}

We reclassified the selected studies into five types based on the study design, which could differ from the design indicated by the authors:

1a. prospective cohort studies (with reference group).

1b. retrospective cohort studies (with reference group).

2a. prospective exposed groups (without reference group).

2b. retrospective exposed groups (without reference group).

3. case-control studies.

We analysed studies that included stillbirths, fetal deaths, and pregnancy terminations ('all births') and studies describing only live births both separately and in combination.

\subsection{Data Analysis}

Two authors (JJ and EG) reclassified all specific congenital anomalies into the congenital anomaly subgroups of the European Surveillance of Congenital Anomalies (EUROCAT) [10]. Only major anomalies were included in the 


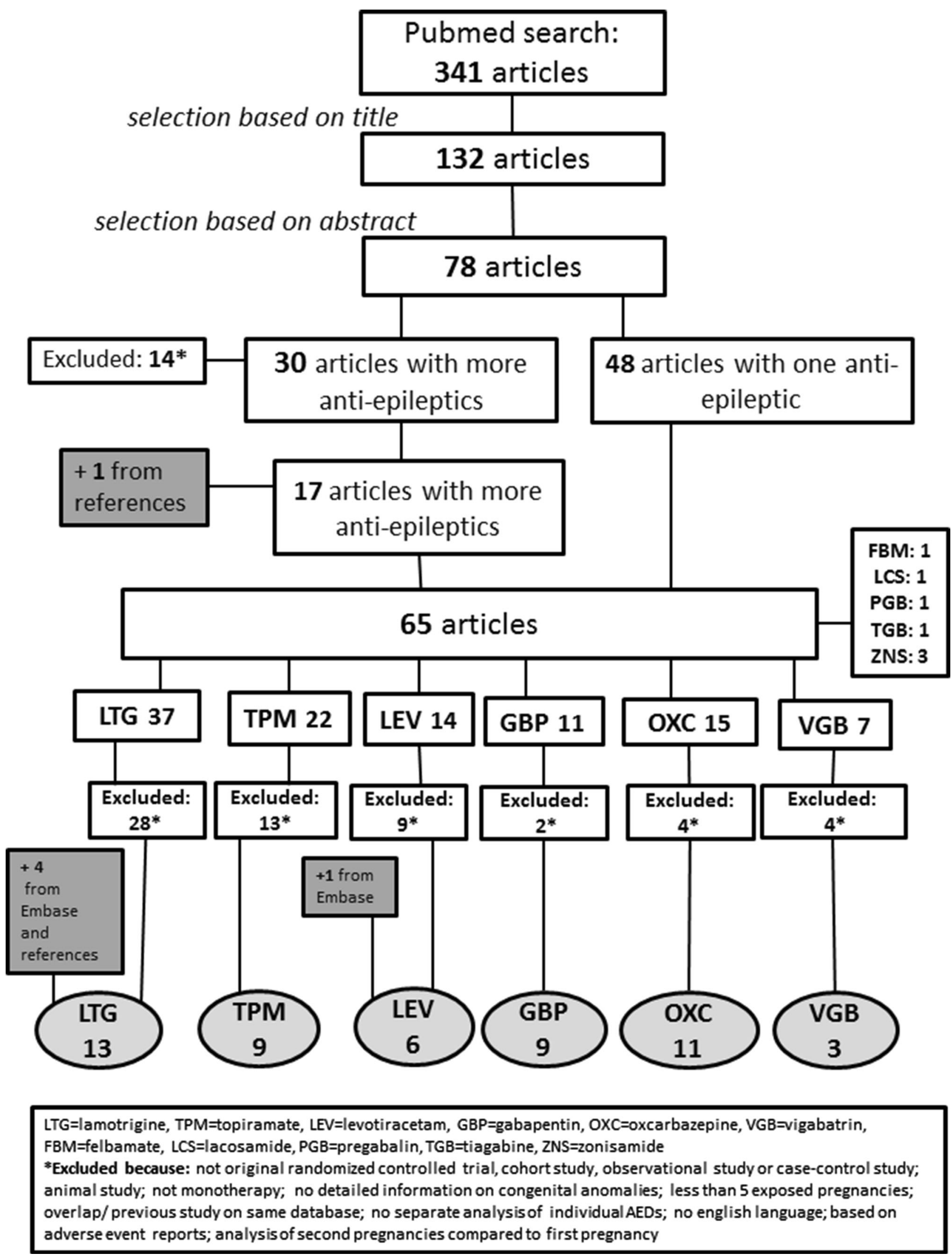

Fig. 1 Selection process for articles included in the review 
analysis. When no information was provided on specific congenital anomalies, we followed the definition of major congenital anomalies in the article.

We calculated the overall major anomaly rate for every AED, for which cohort studies or studies with exposed groups $(1 \mathrm{a}, 1 \mathrm{~b}, 2 \mathrm{a}$, and $2 \mathrm{~b})$ were selected, using the number of fetuses as the denominator. If the analysis in an article was based on pregnancies, and the exact number of fetuses was unknown, we used the number of pregnancies, assuming that multiple pregnancies are rare and that this difference would not greatly influence the results.

We calculated the prevalence of any specific congenital anomaly subgroup from studies with sufficiently detailed information.

The prevalence of specific anomalies was compared with the prevalence of the anomaly subgroup in the EUROCAT AED database, which covers 10,061,059 births from 21 regions in Europe (1995-2011) and has been described previously $[11,12]$. We excluded registrations from this database with maternal AED exposure, maternal epilepsy, or chromosomal anomalies. A significantly higher prevalence of a specific congenital anomaly, based on three or more fetuses with anomalies among fetuses exposed to newer AEDs compared with the prevalence in the reference database, was considered a signal. This procedure is based on the method used by Jentink et al. [12] (see the Electronic Supplementary Material [ESM], Sect. 2), who searched for signals for investigation in a case-control study and noted that it is preferable to detect only the strongest signals. If one or two fetuses with a specific congenital anomaly are found within all the literature searched, it could be that coincidence may have played a major role. We decided to draw the limit at three or more fetuses with a specific anomaly. The case-control studies found were not included in the analysis but were summarized separately.

\subsection{Statistical Analysis}

We calculated the anomaly rate and confidence interval using the method described by Newcombe [13]: the Wilson 'score' method. To compare the prevalence of specific congenital anomalies in the fetuses exposed to newer AEDs with those in the reference database, we used Pearson's Chi-squared test and Yates' continuity correction. We used Microsoft ${ }^{\circledR}$ Office Excel (Microsoft ${ }^{\circledR}$ Corp., Redmond, WA, USA) and R version 3.1.0 (Free Software, Free Software Foundation, Boston, MA, USA) software for the data analysis. A $p$ value $<0.05$ was considered statistically significant.

\section{Results}

\subsection{General Results}

We selected 341 articles from PubMed. In addition, six articles from EMBASE and references were added (Fig. 1; grey background). The 30 selected studies were categorized per AED and study design, as shown in Table 1. We found no randomized controlled trials.

Table 2 shows an overview of the cohort- and exposed group studies found, with the numbers of fetuses or pregnancies exposed to lamotrigine, topiramate, levetiracetam, gabapentin, or oxcarbazepine and the percentage of major congenital anomalies. Vajda et al. [14] provided no information on whether the congenital anomalies were major or minor, so we counted the total number of congenital anomalies. One study [15] included only live births.

Two studies were based on international pregnancy registries and were analysed separately: Cunnington et al. [16] used the International Lamotrigine Pregnancy Registry, with information on specific congenital anomalies of fetuses exposed to lamotrigine; Tomson et al. [17] based their study on the European Registry of Antiepileptic Drugs and Pregnancy (EURAP), a registry of pregnancies exposed to all types of AEDs in 42 countries. The latter provided only information about some congenital anomaly subgroups from pregnancies exposed to newer AEDs. Whether the congenital anomalies were major or minor was not defined, so we used the total number of congenital anomalies to calculate the anomaly rate.

No congenital anomaly was found in fetuses exposed to felbamate, lacosamide, pregabalin, tiagabine, vigabatrin, or zonisamide, but the number of exposed fetuses was very low (1 to 10$)$, except for zonisamide $(n=97)$. No studies describing exposed pregnancies were found for eslicarbazepine, retigabine, rufinamide, or stiripentol.

The studies based on the International Lamotrigine Pregnancy Registry [16] and EURAP registry [17], both international pregnancy registries, were analysed separately because they involve data from, respectively, 43 and 42 countries and might overlap with the other studies.

Table 3 shows the prevalence of specific congenital anomaly subgroups according to the EUROCAT classifications based on the studies with information on specific congenital anomalies compared with that in the reference (EUROCAT AED) database. Two studies [18, 19] with information on congenital anomaly subgroups on a higher level (e.g., 'nervous system' or 'cardiovascular heart defects') among lamotrigine-exposed pregnancies were included in the calculation of the prevalence of these higher-level subgroups, using another denominator. 
Table 1 Number of included studies, stratified by anti-epileptic drug and study design

\begin{tabular}{|c|c|c|c|c|c|c|c|c|c|c|c|c|}
\hline Study design & LTG & TPM & LEV & GBP & $\mathrm{OXC}$ & FBM & LCS & TGB & VGB & ZNS & PGB & Total \\
\hline $\begin{array}{l}\text { 1a: prospective } \\
\text { cohort study }\end{array}$ & $\begin{array}{l}3[14,20, \\
24]\end{array}$ & $\begin{array}{l}4[14, \\
20,27, \\
28]\end{array}$ & $\begin{array}{c}2[14, \\
20]\end{array}$ & $\begin{array}{c}4[14,20, \\
32,35]\end{array}$ & $\begin{array}{c}4[14,20, \\
38,40]\end{array}$ & 0 & 0 & ${ }^{1}$ & ${ }^{1}$ & ${ }^{1}[20]$ & 0 & 20 \\
\hline $\begin{array}{l}\text { 1b: retrospective } \\
\text { cohort study }\end{array}$ & $2[19,21]$ & $\begin{array}{c}2[19, \\
21]\end{array}$ & 1 [19] & $1[21]$ & $\begin{array}{l}3[19,21, \\
39]\end{array}$ & 0 & 0 & 0 & 0 & 0 & 0 & 9 \\
\hline $\begin{array}{l}\text { 2a: prospective } \\
\text { exposed group }\end{array}$ & $\begin{array}{c}5[16-18, \\
22,23]\end{array}$ & $1[17]$ & $\begin{array}{c}2[17, \\
30]\end{array}$ & $2[17,33]$ & $\begin{array}{l}3[17,36, \\
37]\end{array}$ & ${ }^{1}$ & ${ }^{1}$ & 0 & ${ }^{1}$ & ${ }^{1}$ & ${ }^{1}$ & 18 \\
\hline $\begin{array}{l}\text { 2b: retrospective } \\
\text { exposed group }\end{array}$ & $1[15]$ & $1[15]$ & $1[31]$ & $2[15,34]$ & $1[15]$ & 0 & 0 & 0 & ${ }^{1}[15]$ & 1 & 0 & 8 \\
\hline 3: case-control study & $2[25,26]$ & $1[29]$ & 0 & 0 & 0 & 0 & 0 & 0 & 0 & 0 & 0 & 3 \\
\hline Total & 13 & 9 & 6 & 9 & 11 & 1 & 1 & 1 & 3 & 3 & 1 & $58^{\mathrm{a}}$ \\
\hline
\end{tabular}

FBM felbamate, GBP gabapentin, $L C S$ lacosamide, $L E V$ levetiracetam, $L T G$ lamotrigine, $O X C$ oxcarbazepine, $P G B$ pregabalin, $T G B$ tiagabine, $T P M$ topiramate, $V G B$ vigabatrin, $Z N S$ zonisamide

a 30 studies, of which six are counted several times into different drug groups: two prospective cohort studies: one including six individual antiepileptics and one including seven individual antiepileptics; two retrospective cohort studies: one including four and one including three individual antiepileptics; one prospective exposed group including nine individual antiepileptics; one retrospective exposed group including five individual antiepileptics

Table 4 is a summary of the odds ratios (ORs) found in the three selected case-control studies. These case-control studies used databases of pregnancies with congenital anomalies and the (adjusted) ORs of specific congenital anomalies was determined comparing use and no use of specific AEDs.

\subsection{Lamotrigine}

A total of 5197 fetuses exposed to lamotrigine monotherapy in the first trimester were described, 123 of which had major congenital anomalies. The anomaly rate was $2.3 \%$ in the studies with all births; inclusion of the study with only live births [15] changed the rate to $2.4 \%$ (Table 2). In the International Lamotrigine Pregnancy Registry [16], the anomaly rate was $1.7 \%$. The study based on the EURAP registry [17] reported an anomaly rate of $2.9 \%$.

The analysis of specific congenital anomalies is based on seven studies [18-24]. The data from the International Lamotrigine Pregnancy Registry [16] were also compared with the reference database (Table 3). For most specific congenital anomalies, the prevalence among fetuses exposed to lamotrigine monotherapy in the first trimester is comparable with the prevalence of the reference database. In the analysis based on the International Lamotrigine Pregnancy Registry [16], the specific congenital anomalies anencephaly and transposition of great vessels were significantly more prevalent than in the reference database, based on three or more fetuses with anomalies (1.77 vs. $0.20 / 1000, p<0.001 ; 1.77$ vs. $0.30 / 1000, p=0.005$, respectively).

In two case-control studies by Dolk et al. [25] (casemalformed-control study) and Werler et al. [26], no association between the use of lamotrigine in pregnancy and congenital anomalies was found (Table 4).

\subsection{Topiramate}

In seven studies, 553 fetuses were exposed to topiramate, 20 of whom had congenital anomalies (Table 2). The anomaly rate was $3.6 \%$ without and with the study including only live births [15], which was higher than for the other newer AEDs. The study based on the EURAP registry [17] reported an anomaly rate of $6.8 \%$. The prevalence of specific congenital anomalies was calculated from six studies [14, 15, 20, 21, 27, 28]. Two specific congenital anomalies were significantly more prevalent, based on three or more fetuses with anomalies, in the fetuses exposed to topiramate than in the reference database: cleft lip with or without cleft palate (13.86 vs. $0.84 /$ $1000, p<0.001)$ and hypospadias (7.92 vs. $1.53 / 1000$, $p=0.002)$ (Table 3).

The case-control study by Margulis et al. [29] found an OR $>1$ for cleft lip with/without palate for pregnancies exposed to topiramate.

\subsection{Levetiracetam}

The major congenital anomaly rate of fetuses exposed to levetiracetam monotherapy in the first trimester was $1.1 \%$ (11 of 957) and $1.6 \%$ in the EURAP study [17]. Three studies provided specific information on congenital anomalies [20, 30, 31]. Based on three or more cases among fetuses exposed to levetiracetam, no congenital anomaly was more prevalent than in the reference database (Table 3). 
Table 2 Overview of the studies including fetuses/pregnancies exposed to monotherapy with antiepileptic drugs in the first trimester

\begin{tabular}{|c|c|c|c|c|c|c|}
\hline Study & Country & Birth years & $\begin{array}{l}\text { Fetuses/ } \\
\text { pregnancies }\end{array}$ & No. exposed & $\begin{array}{l}\text { No. of congenital } \\
\text { anomalies }^{\mathrm{a}}\end{array}$ & Drug \\
\hline \multirow[t]{3}{*}{ Mawer et al. $[21]^{\mathrm{b}}$} & UK & 2000-2006 & $\mathrm{F}$ & 37 & 2 & Lamotrigine \\
\hline & & & & 3 & 0 & Topiramate \\
\hline & & & & 2 & 0 & Gabapentin \\
\hline \multirow[t]{5}{*}{ Hernández-Díaz et al. [20] } & USA & 1997-2011 & $\mathrm{F}$ & 1562 & $24^{\mathrm{c}}$ & Lamotrigine \\
\hline & & & & 359 & $13^{\mathrm{c}}$ & Topiramate \\
\hline & & & & 450 & $6^{\mathrm{c}}$ & Levetiracetam \\
\hline & & & & 145 & 1 & Gabapentin \\
\hline & & & & 182 & 4 & Oxcarbazepine \\
\hline \multirow[t]{5}{*}{ Vajda et al. $[14]^{\mathrm{d}}$} & Australia & 1998-2013 & $\mathrm{F}$ & 310 & 15 & Lamotrigine \\
\hline & & & & 43 & 1 & Topiramate \\
\hline & & & & 83 & 2 & Levetiracetam \\
\hline & & & & 10 & 0 & Gabapentin \\
\hline & & & & 17 & 1 & Oxcarbazepine \\
\hline \multirow[t]{4}{*}{ Veiby et al. [19] } & Norway & 1999-2011 & $\mathrm{F}$ & 833 & 28 & Lamotrigine \\
\hline & & & & 48 & 2 & Topiramate \\
\hline & & & & 118 & 2 & Levetiracetam \\
\hline & & & & 57 & 1 & Oxcarbazepine \\
\hline Meador [22] & USA/UK & 1999-2004 & $\mathrm{F}$ & 98 & 1 & Lamotrigine \\
\hline Miskov et al. [23] & Croatia & 2003-2008 & $\mathrm{P}$ & 23 & 0 & Lamotrigine \\
\hline Cassina et al. [24] & Italy & 2000-2008 & $\mathrm{P}$ & 46 & 0 & Lamotrigine \\
\hline Campbell et al. [18] & UK/Ireland & 1996-2012 & $\mathrm{F}$ & 2198 & 49 & Lamotrigine \\
\hline Hunt et al. [27] & UK & 1996-2007 & $\mathrm{F}$ & 70 & 3 & Topiramate \\
\hline Ornoy et al. [28] & Israel & 1996-2006 & $\mathrm{F}$ & 29 & 1 & Topiramate \\
\hline Ten Berg et al. [31] & The Netherlands & - & $\mathrm{F}$ & 2 & 0 & Levetiracetam \\
\hline Mawhinney et al. [30] & UK/Ireland & 2000-2011 & $\mathrm{P}$ & 304 & $1^{\mathrm{c}}$ & Levetiracetam \\
\hline Montouris [34] & USA & - & $\mathrm{P}$ & 17 & $0^{\mathrm{c}}$ & Gabapentin \\
\hline Morrow et al. [35] & UK & 1996-2005 & $\mathrm{P}$ & 31 & 1 & Gabapentin \\
\hline Guttusso et al. [33] & USA & 2008-2009 & $\mathrm{F}$ & 7 & 2 & Gabapentin \\
\hline Fujii et al. [32] & Diverse $^{\mathrm{e}}$ & - & $\mathrm{P}$ & 36 & 0 & Gabapentin \\
\hline Samren et al. [41] & The Netherlands & 1972-1994 & $\mathrm{F}$ & 2 & 0 & Oxcarbazepine \\
\hline Hvas et al. [40] & Denmark & 1989-1997 & $\mathrm{F}$ & 7 & 0 & Oxcarbazepine \\
\hline Kaaja et al. [37] ${ }^{\mathrm{f}}$ & Finland & 1990-1998 & $\mathrm{F}$ & 9 & 1 & Oxcarbazepine \\
\hline Meischenguiser et al. [36] & Argentina & 1995-2002 & $\mathrm{F}$ & 35 & 0 & Oxcarbazepine \\
\hline Artama et al. [39] & Finland & 1991-2000 & $\mathrm{F}$ & 99 & 1 & Oxcarbazepine \\
\hline Viinikainen et al. $[38]^{\mathrm{f}}$ & Finland & 1989-2000 & $\mathrm{F}$ & 2 & 0 & Oxcarbazepine \\
\hline \multirow[t]{10}{*}{ Total $^{\mathrm{g}}$} & & & & 5107 & 119 & Lamotrigine \\
\hline & & & & {$[2.3(2.0-2.8)]$} & & \\
\hline & & & & 552 & 20 & Topiramate \\
\hline & & & & {$[3.6(2.3-5.5)]$} & & \\
\hline & & & & 957 & 11 & Levetiracetam \\
\hline & & & & {$[1.1(0.6-2.0)]$} & & \\
\hline & & & & 248 & 4 & Gabapentin \\
\hline & & & & {$[1.6(0.6-4.0)]$} & & \\
\hline & & & & 410 & 8 & Oxcarbazepine \\
\hline & & & & {$[2.0(0.9-3.8)]$} & & \\
\hline
\end{tabular}


Table 2 continued

\begin{tabular}{|c|c|c|c|c|c|c|}
\hline Study & Country & Birth years & $\begin{array}{l}\text { Fetuses/ } \\
\text { pregnancies }\end{array}$ & No. exposed & $\begin{array}{l}\text { No. of congenital } \\
\text { anomalies }^{\mathrm{a}}\end{array}$ & Drug \\
\hline \multicolumn{7}{|c|}{ Studies including only live births } \\
\hline \multirow[t]{4}{*}{ Wide [15] } & \multirow[t]{4}{*}{ Sweden } & \multirow[t]{4}{*}{ 1995-2001 } & \multirow[t]{14}{*}{$\mathrm{F}$} & 90 & 4 & Lamotrigine \\
\hline & & & & 1 & 0 & Topiramate \\
\hline & & & & 18 & 0 & Gabapentin \\
\hline & & & & 4 & 0 & Oxcarbazepine \\
\hline \multirow{10}{*}{$\begin{array}{l}\text { Total (studies including } \\
\text { only live births) }\end{array}$} & & & & 5197 & 123 & Lamotrigine \\
\hline & & & & {$[2.4(2.0-2.8)]$} & & \\
\hline & & & & 553 & 20 & Topiramate \\
\hline & & & & {$[3.6(2.4-5.5)]$} & & \\
\hline & & & & 957 & 11 & Levetiracetam \\
\hline & & & & {$[1.1(0.6-2.0)]$} & & \\
\hline & & & & 266 & 4 & Gabapentin \\
\hline & & & & {$[1.5(0.6-2.0)]$} & & \\
\hline & & & & 414 & 8 & Oxcarbazepine \\
\hline & & & & {$[1.9(1.0-3.8)]$} & & \\
\hline \multicolumn{7}{|c|}{ International Pregnancy Registry Studies } \\
\hline Cunnington et al. $[16]^{\mathrm{g}}$ & $\begin{array}{l}\text { International Lamotrigine } \\
\text { Pregnancy Registry }\end{array}$ & 1992-2010 & $\mathrm{F}$ & $\begin{array}{l}1699 \\
{[1.7(1.2-2.4)]}\end{array}$ & 29 & Lamotrigine \\
\hline \multirow[t]{10}{*}{ Tomson et al. $[17]^{\mathrm{g}}$} & \multirow[t]{10}{*}{ EURAP Registry $^{\mathrm{h}}$} & \multirow[t]{10}{*}{ 1999-2010 } & \multirow[t]{10}{*}{$\mathrm{F}$} & 1280 & 37 & Lamotrigine \\
\hline & & & & {$[2.9(2.1-4.0)]$} & & \\
\hline & & & & 73 & 5 & Topiramate \\
\hline & & & & {$[6.8(3.0-15.0)]$} & & \\
\hline & & & & 126 & 2 & Levetiracetam \\
\hline & & & & [1.6 (0.4-5.6)] & & \\
\hline & & & & 23 & 0 & Gabapentin \\
\hline & & & & {$[\mathrm{NA}]$} & & \\
\hline & & & & 184 & 6 & Oxcarbazepine \\
\hline & & & & [3.3 (1.5-6.9)] & & \\
\hline
\end{tabular}

EURAP European Registry of Antiepileptic Drugs and Pregnancy, $F$ fetus, $N A$ not applicable, $P$ pregnancy

${ }^{a}$ Number of fetuses with one or more major congenital anomalies

b Possible overlap with Hernández-Díaz et al

${ }^{c}$ Excludes fetuses with minor anomalies

${ }^{\mathrm{d}}$ Major and minor anomalies not separated

e Canada, France, England, Italy, Korea

${ }^{\mathrm{f}}$ Possible overlap with Artama et al

g Data are presented as [major congenital anomaly rate \% (95\% confidence interval)]

${ }^{\mathrm{h}}$ Includes major and minor congenital malformation

\subsection{Gabapentin}

Four of 266 fetuses exposed to gabapentin in the first trimester had major congenital anomalies (Table 2). Congenital anomalies were not found in the study that included only live births [15] or in the EURAP study [17]. The overall anomaly rate was $1.5 \%$. We used seven studies with detailed information [15, 21, 28, 32-35] for the analysis of specific major congenital anomalies. No significant differences were found between the prevalence of the specific congenital anomalies and the prevalence in the reference database (Table 3 ).

\subsection{Oxcarbazepine}

Eight of 414 fetuses exposed to oxcarbazepine in the first trimester of pregnancy had major congenital anomalies. The anomaly rate was $2.0 \%$ of the studies with all births and $1.9 \%$ including the study with live births [15]. The EURAP study [17] had an anomaly rate of $3.3 \%$. The prevalence of 
Table 3 Prevalence of specific anomaly subgroups of fetuses/pregnancies exposed to lamotrigine, topiramate, levetiracetam, gabapentin, or oxcarbazepine compared with the reference database

\begin{tabular}{|c|c|c|c|c|c|c|c|}
\hline \multirow[t]{2}{*}{ Congenital anomaly } & \multicolumn{6}{|c|}{ Prevalence (no. cases, no. studies) } & \multirow{2}{*}{$\begin{array}{l}\text { Prevalence (no. } \\
\text { cases) } \\
\text { EUROCAT AED } \\
\text { database } \\
\text { (reference) } \\
(N=10,061,059)\end{array}$} \\
\hline & Lamotrigine & $\begin{array}{l}\text { Lamotrigine } \\
\text { International } \\
\text { Pregnancy } \\
\text { Registry [16] }\end{array}$ & Topiramate & Levetiracetam & Gabapentin & Oxcarbazepine & \\
\hline Nervous system & $2.08(10,4)$ & $2.35(4,1)$ & & $1.32(1,1)$ & $8.26(1,1)$ & & $1.67(16,752)$ \\
\hline $\begin{array}{l}\text { Unspecified [16, 20, 21, } \\
33]\end{array}$ & NA $(2,2)$ & NA $(1,1)$ & & & NA $(1,1)$ & & \\
\hline $\begin{array}{l}\text { Anencephalus and } \\
\text { similar }[16,20]\end{array}$ & $0.57(1,1)$ & $1.77^{\mathrm{a}}(3,1)$ & & $1.32(1,1)$ & & & 0.2 (1977) \\
\hline Spina bifida [20] & $0.57(1,1)$ & & & & & & $0.4(4005)$ \\
\hline Hydrocephalus [20] & $0.57(1,1)$ & & & & & & $0.39(3946)$ \\
\hline $\begin{array}{l}\text { Arhinencephaly/ } \\
\text { holoprosencephaly [20] }\end{array}$ & $1.13(2,1)$ & & & & & & $0.06(565)$ \\
\hline Eye & & & & $1.32(1,1)$ & & & $0.38(3820)$ \\
\hline Congenital cataract [20] & & & & $1.32(1,1)$ & & & $0.09(943)$ \\
\hline $\mathrm{CHD}^{\mathrm{b}}$ & $7.50(36,4)$ & $5.30(9,1)$ & $5.94(3,2)$ & $1.32(1,1)$ & $8.26(1,1)$ & & $6.71(67,535)$ \\
\hline Unspecified [28] & & & $\mathrm{NA}(1,1)$ & & & & \\
\hline $\begin{array}{l}\text { Transposition of great } \\
\text { vessels }[16,20]\end{array}$ & $0.57(1,1)$ & $1.77^{\mathrm{a}}(3,1)$ & & & & & $0.30(2988)$ \\
\hline VSD $[16,20,22,35]$ & $0.57(1,1)$ & $1.77(3,1)$ & $1.98(1,1)$ & $1.32(1,1)$ & $8.26(1,1)$ & & $3.34(33,642)$ \\
\hline ASD [20] & $2.27(4,1)$ & & $1.98(1,1)$ & & & & $1.94(19,484)$ \\
\hline Tetralogy of Fallot [16] & & $0.59(1,1)$ & & & & & $0.24(2416)$ \\
\hline $\begin{array}{l}\text { Pulmonary valve } \\
\text { stenosis }[16,20]\end{array}$ & $0.57(1,1)$ & $0.59(1,1)$ & & & & & $0.34(3450)$ \\
\hline $\begin{array}{l}\text { Pulmonary valve atresia } \\
\text { [20] }\end{array}$ & $0.57(1,1)$ & & & & & & $0.08(853)$ \\
\hline $\begin{array}{l}\text { Hypoplastic left heart } \\
\text { [16] }\end{array}$ & & $1.18^{\mathrm{a}}(2,1)$ & & & & & 0.19 (1910) \\
\hline $\begin{array}{l}\text { Patent ductus arteriosus } \\
{[20]}\end{array}$ & & & $1.98(1,1)$ & & & & $0.33(3330)$ \\
\hline Respiratory & $0.63(3,2)$ & & $1.98(1,1)$ & & & $2.94(1,1)$ & $0.44(4406)$ \\
\hline Unspecified [20] & & & NA $(1,1)$ & & & & \\
\hline Choanal atresia [20] & $0.57(1,1)$ & & & & & & $0.06(569)$ \\
\hline $\begin{array}{l}\text { Cystic adenomatous } \\
\text { malformation of lung } \\
{[20]}\end{array}$ & & & & & & $2.94^{\mathrm{a}}(1,1)$ & $0.03(338)$ \\
\hline Orofacial clefts & $2.08(10,3)$ & $1.18(2,1)$ & $\begin{array}{l}13.86^{\mathrm{a}}(7, \\
2)\end{array}$ & & & $2.94(1,1)$ & $1.36(13,720)$ \\
\hline $\begin{array}{l}\text { Cleft lip with or without } \\
\text { palate }[16,20,27]\end{array}$ & $2.27(4,1)$ & $0.59(1,1)$ & $\begin{array}{l}13.86^{\mathrm{a}}(7, \\
2)\end{array}$ & & & & $0.84(8470)$ \\
\hline Cleft palate $[16,20]$ & $1,70(3,1)$ & $0.59(1,1)$ & & & & $2.94(1,1)$ & $0.52(5247)$ \\
\hline Digestive system & $2.50(12,2)$ & $1.77(3,1)$ & $1.98(1,1)$ & $3.97(3,2)$ & & & $1.45(14,604)$ \\
\hline $\begin{array}{l}\text { Oesophageal atresia } \\
\text { with or without trachea } \\
\text { oesophageal fistula [30] }\end{array}$ & & & & $1.32(1,1)$ & & & $0.21(2138)$ \\
\hline $\begin{array}{l}\text { Duodenal atresia or } \\
\text { stenosis [20] }\end{array}$ & & & $1.98^{\mathrm{a}}(1,1)$ & & & & 0.08 \\
\hline $\begin{array}{l}\text { Atresia or stenosis of } \\
\text { other parts of small } \\
\text { intestine [20] }\end{array}$ & & & & $1.32^{\mathrm{a}}(1,1)$ & & & $0.06(593)$ \\
\hline $\begin{array}{l}\text { Ano-rectal atresia and } \\
\text { stenosis }[16,20]\end{array}$ & & $0.59(1,1)$ & & $1.32(1,1)$ & & & $0.94(2668)$ \\
\hline
\end{tabular}


Table 3 continued

\begin{tabular}{|c|c|c|c|c|c|c|c|}
\hline \multirow[t]{2}{*}{ Congenital anomaly } & \multicolumn{6}{|c|}{ Prevalence (no. cases, no. studies) } & \multirow{2}{*}{$\begin{array}{l}\text { Prevalence (no. } \\
\text { cases) } \\
\text { EUROCAT AED } \\
\text { database } \\
\text { (reference) } \\
(N=10,061,059)\end{array}$} \\
\hline & Lamotrigine & $\begin{array}{l}\text { Lamotrigine } \\
\text { International } \\
\text { Pregnancy } \\
\text { Registry [16] }\end{array}$ & Topiramate & Levetiracetam & Gabapentin & Oxcarbazepine & \\
\hline $\begin{array}{l}\text { Diaphragmatic hernia } \\
\text { [16] }\end{array}$ & & $1.18^{\mathrm{a}}(2,1)$ & & & & & $0.20(1985)$ \\
\hline Urinary & $0.57(1,1)$ & $1.77(3.1)$ & $1.98(1,1)$ & $1.32(1,1)$ & $8.26(1,1)$ & $2.94(1,1)$ & $2.45(24,649)$ \\
\hline Unspecified [20] & & & NA $(1,1)$ & & & & \\
\hline Renal dysplasia [20] & & & & $1.32(1,1)$ & & & $0.23(2364)$ \\
\hline $\begin{array}{l}\text { Congenital } \\
\text { hydronephrosis [16, 33, } \\
37]\end{array}$ & & $1.77(3,1)$ & & & $8.26(1,1)$ & $2.94(1,1)$ & $0.85(8519)$ \\
\hline $\begin{array}{l}\text { PUV and/or prune belly } \\
\text { [20] }\end{array}$ & $0.57(1,1)$ & & & & & & $0.08(793)$ \\
\hline Genital & $1.92(5,2)$ & $1.92(5,2)$ & $7.92^{\mathrm{a}}(4,2)$ & & & $5.88(2,2)$ & $1.80(18,115)$ \\
\hline Unspecified [39] & & & & & & NA $(1,1)$ & \\
\hline Hypospadias $[20,28]^{\mathrm{c}}$ & $0.57(1,1)$ & $1.18(2,1)$ & $7.92^{\mathrm{a}}(4,2)$ & & & $2.94(1,1)$ & $1.53(15,395)$ \\
\hline Limb & $2.27(4,2)$ & $3.53(6,1)$ & $7.92(4,1)$ & & & $2.94(1,1)$ & $3.94(39,652)$ \\
\hline Limb reduction [20] & $1.13(2,1)$ & & $1.98(1,1)$ & & & & $0.51(5162)$ \\
\hline Club foot $[16,20,21]$ & $0.57(1,1)$ & $1.77(3,1)$ & $1.98(1,1)$ & & & & $0.90(9042)$ \\
\hline $\begin{array}{l}\text { Hip dislocation and/or } \\
\text { dysplasia }[16,20]\end{array}$ & & $0.59(1,1)$ & & & & $2.94(1,1)$ & $0.58(5814)$ \\
\hline Polydactyly $[16,20]$ & $0.57(1,1)$ & $1.18(2,1)$ & $1.98(1,1)$ & & & & $0.87(8789)$ \\
\hline Syndactyly [20] & & & $1.98(1,1)$ & & & & $0.46(4660)$ \\
\hline \multicolumn{8}{|l|}{ Other } \\
\hline Craniosynostosis [20] & $0.57(1,1)$ & & & & & & $0.17(1737)$ \\
\hline $\begin{array}{l}\text { Congenital skin } \\
\text { disorder [16] }\end{array}$ & & $0.59(1,1)$ & & & & & $0.22(2232)$ \\
\hline
\end{tabular}

$A E D$ anti-epileptic drug, $A S D$ atrial septal defect, $C H D$ congenital heart defect, EUROCAT European Surveillance of Congenital Anomalies, NA not applicable, $P U V$ posterior urethral valve, VSD ventricular septal defect

a Significant difference

b One infant with transposition of great vessels, ASD, and pulmonary valve atresia, one infant with transposition of great vessels and tetralogy of Fallot, and one infant with VSD and ASD were counted once within the total number of CHD

c The only anomaly of topiramate monotherapy in the study by Vajda et al. [14] was hypospadias according to a previous article [46]

specific congenital anomalies was calculated by including eight studies [15, 20, 36-41] (Table 3). Based on three or more cases, no higher prevalence of specific congenital anomalies than in the reference database was found.

\section{Discussion}

In this literature review, we found 30 articles that met our inclusion criteria. The total major anomaly rate of fetuses exposed to topiramate was slightly higher than of those exposed to the other newer AEDs. The anomaly rate for the EURAP study [17] was higher, probably because minor anomalies were included.
No congenital anomalies were found among pregnancies exposed to felbamate, lacosamide, pregabalin, tiagabine, vigabatrin, or zonisamide. Because the number of observations is very low, no conclusions could be drawn about the risk of congenital anomalies for pregnancies exposed to these AEDs. No articles on congenital anomalies were found in relation to the use of eslicarbazepine, retigabine, rufinamide, or stiripentol.

Four signals of possible associations of specific congenital anomalies and use of newer AED monotherapy in the first trimester of pregnancy were detected: two related to lamotrigine (anencephaly and transposition of great vessels) and two related to topiramate (cleft lip with or without cleft palate and hypospadias). 
Table 4 Summary of the selected case-control studies

\begin{tabular}{|c|c|c|c|c|}
\hline Study & AED & Database & Congenital anomaly & OR (95\% CI) (use/no use) \\
\hline \multirow[t]{4}{*}{ Dolk et al. [25] } & \multirow[t]{4}{*}{ Lamotrigine } & \multirow[t]{4}{*}{ EUROCAT } & Isolated orofacial clefts & $0.80(0.11-2.85)$ \\
\hline & & & Isolated and multiple orofacial clefts & $0.67(0.10-2.85)$ \\
\hline & & & Isolated cleft palate & $1.01(0.03-5.57)$ \\
\hline & & & Isolated and multiple cleft palate & $0.79(0.03-4.35)$ \\
\hline \multirow[t]{4}{*}{ Werler et al. [26] } & \multirow[t]{4}{*}{ Lamotrigine } & \multirow[t]{4}{*}{ NBDPS } & Oral clefts & $4.3(0.71-26.2)$ \\
\hline & & & Heart defects & $1.7(0.31-9.3)$ \\
\hline & & & Hypospadias & $2.7(0.17-44.0)$ \\
\hline & & & Other & $1.2(0.17-8.4)$ \\
\hline \multirow[t]{6}{*}{ Margulis et al. [29] } & \multirow[t]{6}{*}{ Topiramate } & \multirow[t]{2}{*}{ BDS } & Any major & $1.22(0.19-13.01)$ \\
\hline & & & Cleft lip with/without palate & $10.13(1.09-129.21)$ \\
\hline & & \multirow[t]{2}{*}{ NBDPS } & Any major & $0.92(0.26-4.06)$ \\
\hline & & & Cleft lip with/without palate & $3.63(0.66-20.00)$ \\
\hline & & \multirow[t]{2}{*}{ Pooled } & Any major & $1.01(0.37-3.22)$ \\
\hline & & & Cleft lip with/without palate & $5.36(1.49-20.07)$ \\
\hline
\end{tabular}

$A E D$ anti-epileptic drug, BDS Slone Epidemiology Center Birth Defects Study, CI confidence interval, EUROCAT European Surveillance of Congenital Anomalies, NBPDS National Birth Defects Prevention Study, OR odds ratio, pooled indicates that these databases are pooled

\subsection{Lamotrigine}

Two signals were found in the analysis of the study based on the International Lamotrigine Pregnancy Registry [16]. The first signal was a significantly higher prevalence of anencephaly (three cases) compared with the reference database. Anencephaly was also found in the analysis of the other combined studies but not significantly more frequently than in the reference database. The high prevalence of anencephaly was mentioned in the discussion of the study [16], but an association was not concluded because this finding was not supported by other studies.

The prevalence of transposition of great vessels is higher in the International Lamotrigine Pregnancy Registry [16] than in the reference database. In the combined analysis, transposition of great vessels was found; however, the prevalence was similar to that in the reference database. Cunnington et al. [16] stated that several cases of severe cardiac defects were found but that other studies fail to support an increased frequency of cardiac heart defects among fetuses exposed to lamotrigine in utero.

In this review, we did not find an association between lamotrigine and orofacial clefts, which was one of the conclusions in a previous review [42]. This conclusion was based on studies from the North American AED Pregnancy Registry, which found an increased frequency of isolated cleft palate in infants exposed to lamotrigine during pregnancy compared with the reference population [20,43]. The case-malformed-control study by Dolk et al. [25] did not find an association between lamotrigine and orofacial clefts. A letter responding to the review stated that the comparison group in the North American AED Pregnancy
Registry had a low prevalence of orofacial clefts compared with other databases [44]. In the case-control study by Werler et al. [26] in the USA, based on the National Birth Defect Prevention Study, no significant association was found between orofacial clefts and lamotrigine. That we did not find signals of an association between orofacial clefts and lamotrigine exposure in pregnancy, combining all known cohort studies, supports that there is no association between lamotrigine and orofacial clefts.

\subsection{Topiramate}

We found a signal for the congenital anomaly subgroup cleft lip with or without palate in fetuses exposed to topiramate in the first trimester of pregnancy. The prevalence is significantly higher than in the reference database and is based on seven fetuses. Five of these were from the study by Hernández-Díaz et al. (North American AED Pregnancy Registry) [20], and two were from the study by Hunt et al. (UK Epilepsy and Pregnancy Register) [27]. The conclusion of the study by Hernández-Díaz et al. [20] mentioned an association between topiramate monotherapy use in pregnancy and cleft lip. The conclusion of the study by Hunt et al. [27] also mentioned an association between topiramate and orofacial clefts, but their analysis was based on two fetuses with exposure to topiramate as monotherapy and two fetuses with topiramate in combination with other AEDs. The case-control study by Margulis et al. [29], using two North-American databases, found an association between topiramate and cleft lip with or without palate. Mines et al. [45] used four different data sources from the USA and found a higher prevalence of cleft lip in infants 
exposed to topiramate monotherapy than in infants of mothers formerly exposed to topiramate or other AEDs and mothers with similar medical profiles not exposed to topiramate. We conclude there is a strong signal indicating an association between cleft lip and topiramate monotherapy in the first trimester of pregnancy. This signal should be investigated further.

The second signal among fetuses exposed to topiramate monotherapy in the first trimester of pregnancy was a higher risk of hypospadias. Four fetuses with hypospadias were found in three different studies: one from the study by Vajda et al. (Australian Pregnancy Registry) [14], one from the study by Hunt et al. (UK Epilepsy and Pregnancy Register) [27], and two from the study by Hernández-Díaz et al. (North American AED Pregnancy Registry) [20]. Prevalence was significantly higher than in the reference database. Neither Hernández-Díaz et al. [20] nor Hunt et al. [27] mentioned a possible association between hypospadias and the use of topiramate monotherapy in their conclusions. Vajda et al. [28] also did not mention this association in their conclusion. Although they did indicate an association in a previous study [46], it was not for monotherapy.

\subsection{Strengths and Limitations}

A limitation of this review was the small sample size of fetuses exposed to newer AEDs in the observational studies included in the analysis of specific congenital anomalies. The number of exposed fetuses with information for specific congenital anomalies was 505 for topiramate, 756 for levetiracetam, 125 for gabapentin, and 340 for oxcarbazepine. The specific congenital anomaly subgroups with a prevalence of $0.1-5 / 1000$ are most likely missed in these small samples. For lamotrigine, the number of pregnancies with information on specific congenital anomalies was higher ( $n=1766$ and 1699 from the International Lamotrigine Pregnancy Registry [16]), which increased the chance of finding signals.

We found few or no exposed fetuses for six other newer AEDs, except for zonisamide (97 exposed fetuses) and no congenital anomalies. We found no studies on exposure in pregnancy for five newer AEDs and therefore could draw no conclusions on possible associations with specific congenital anomalies.

Another limitation is that enrolment in most of the studies was voluntary. Selection bias may be present because well-educated, motivated, or interested women are more likely to register. It was unknown for most pregnancies whether the women took medication (prescribed or over the counter) other than the AEDs. This possible comedication could also be teratogenic, as could other exposures such as smoking and alcohol use or other unknown circumstances affecting the fetus.
Not all the studies excluded chromosomal anomalies. A few major congenital anomalies from fetuses with chromosomal anomalies may have been included. Because we reclassified the anomalies according to EUROCAT, we consider this will not affect the results.

Differences also exist between the cohorts used in the literature and those in the EUROCAT AED database. The latter contains data from 1995 to 2011, whereas the literature includes studies with data from earlier years, starting from 1972 [41]. Because the data before 1995 are sparse, we consider this unlikely to have affected the conclusions. However, the data from the EUROCAT AED database are mainly from Europe, whereas the literature includes studies from the USA. In particular, one important study with considerable data is from the USA [20]. However, we think the differences between the populations are not large enough to affect the results.

\section{Conclusion}

In this literature review, we sourced information on specific congenital anomalies in fetuses exposed to lamotrigine, topiramate, levetiracetam, gabapentin, or oxcarbazepine monotherapy in the first trimester. Few or no exposed pregnancies and no congenital anomalies were reported for the remaining AEDs. Four signals of possible associations between the use of newer AEDs and specific congenital anomalies were detected: lamotrigine and anencephaly, lamotrigine and transposition of great vessels, topiramate and cleft lip, and topiramate and hypospadias. Of these, only the signal for the association between topiramate and cleft lip with or without cleft palate was considered strong. There might be an association between hypospadias and the use of topiramate in pregnancy, but possibly only for polytherapy. These signals should be investigated further.

Acknowledgments The authors wish to thank Professor Helen Dolk for her comments and advice on the draft manuscript.

Author contributions Josta de Jong performed the analysis for the review and wrote the manuscript. Ester Garne assisted with the categorization of the anomalies and advised on the design of the review and the writing of the manuscript. Hao Wang and Lolkje de Jong-van den Berg were supervisors and advisors during the performance of this review.

\section{Compliance with Ethical Standards}

Funding This study was part of the EUROmediCAT research project (http://www.euromedicat.eu), which is supported by the European Commission under the 7th Framework Programme Grant Agreement No 260598. 
Conflict of interest Josta de Jong, Ester Garne, Lolkje de Jong-van den Berg, and Hao Wang have no conflicts of interest.

Open Access This article is distributed under the terms of the Creative Commons Attribution-NonCommercial 4.0 International License (http://creativecommons.org/licenses/by-nc/4.0/), which permits any noncommercial use, distribution, and reproduction in any medium, provided you give appropriate credit to the original author(s) and the source, provide a link to the Creative Commons license, and indicate if changes were made.

\section{References}

1. Harden CL. Pregnancy and epilepsy. Continuum (Minneap Minn). 2014;20(1 Neurology of Pregnancy):60-79.

2. Harden CL, Meador KJ, Pennell PB, Hauser WA, Gronseth GS, French JA, et al. Management issues for women with epilepsy: focus on pregnancy (an evidence-based review): II. Teratogenesis and perinatal outcomes: Report of the Quality Standards Subcommittee and Therapeutics and Technology Subcommittee of the American Academy of Neurology and the American Epilepsy Society. Epilepsia. 2009;50(5):1237-46.

3. National Institute for Health and Care Excellence (NICE). The epilepsies: the diagnosis and management of the epilepsies in adults and children in primary and secondary care Guidelines CG137: 1.15.3 Pregnancy. 2012; Available at: http://www.nice. org.uk/guidance/cg137/chapter/1-guidance\#women-and-girls-withepilepsy. Accessed 5 Nov 2014.

4. de Groot MC, Schuerch M, de Vries F, Hesse U, Oliva B, Gil M, et al. Antiepileptic drug use in seven electronic health record databases in Europe: a methodologic comparison. Epilepsia. 2014;55(5):666-73.

5. Savica R, Beghi E, Mazzaglia G, Innocenti F, Brignoli O, Cricelli $\mathrm{C}$, et al. Prescribing patterns of antiepileptic drugs in Italy: a nationwide population-based study in the years 2000-2005. Eur J Neurol. 2007;14(12):1317-21.

6. Tsiropoulos I, Gichangi A, Andersen M, Bjerrum L, Gaist D, Hallas J. Trends in utilization of antiepileptic drugs in Denmark. Acta Neurol Scand. 2006;113(6):405-11.

7. Landmark CJ, Fossmark H, Larsson PG, Rytter E, Johannessen SI. Prescription patterns of antiepileptic drugs in patients with epilepsy in a nation-wide population. Epilepsy Res. 2011;95(1-2):51-9.

8. European Medicines Agency. PRAC recommends strengthening the restrictions on the use of valproate in women and girls. 2014; Available at: http://www.ema.europa.eu/ema/index.jsp?curl= pages/medicines/human/referrals/Valproate_and_related_substances /human_referral_prac_000032.jsp\&mid=WC0b01ac05805c516f. Accessed 17 Nov 2014.

9. Charlton RA, Garne E, Wang H, Klungsøyr K, Jordan S, Neville AJ, et al. Antiepileptic drug prescribing before, during and after pregnancy: a study in seven European regions. Pharmacoepidemiol Drug Saf. 2015;24(11):1144-54.

10. EUROCAT. EUROCAT Guide 1.3 and reference documents, instructions for the registration and surveillance of congenital anomalies, September 2005, Available at: http://www.eurocat. ulster.ac.uk/pdf/EUROCAT-Guide-1.3.pdf. Accessed 10 June 2015.

11. Jentink J, Dolk H, Loane MA, Morris JK, Wellesley D, Garne E, et al. Intrauterine exposure to carbamazepine and specific congenital malformations: systematic review and case-control study. BMJ. 2010;2(341):c6581.

12. Jentink J, Loane MA, Dolk H, Barisic I, Garne E, Morris JK, et al. Valproic acid monotherapy in pregnancy and major congenital malformations. N Engl J Med. 2010;362(23):2185-93.
13. Newcombe RG. Two-sided confidence intervals for the single proportion: Comparison of seven methods. Stat Med. 1998;17(8):857-72.

14. Vajda FJ, O'Brien TJ, Lander CM, Graham J, Eadie MJ. The teratogenicity of the newer antiepileptic drugs-an update. Acta Neurol Scand 2014;130(4):234-8

15. Wide K, Winbladh B, Kallen B. Major malformations in infants exposed to antiepileptic drugs in utero, with emphasis on carbamazepine and valproic acid: a nation-wide, population-based register study. Acta Paediatr. 2004;93(2):174-6.

16. Cunnington MC, Weil JG, Messenheimer JA, Ferber S, Yerby M, Tennis P. Final results from 18 years of the International Lamotrigine Pregnancy Registry. Neurology. 2011;76(21): $1817-23$.

17. Tomson T, Battino D, Bonizzoni E, Craig J, Lindhout D, Sabers A, et al. Dose-dependent risk of malformations with antiepileptic drugs: an analysis of data from the EURAP epilepsy and pregnancy registry. Lancet Neurol. 2011;10(7):609-17.

18. Campbell E, Kennedy F, Russell A, Smithson WH, Parsons L, Morrison PJ, et al. Malformation risks of antiepileptic drug monotherapies in pregnancy: updated results from the UK and Ireland Epilepsy and Pregnancy Registers. J Neurol Neurosurg Psychiatry. 2014;85(9):1029-34.

19. Veiby G, Daltveit AK, Engelsen BA, Gilhus NE. Fetal growth restriction and birth defects with newer and older antiepileptic drugs during pregnancy. J Neurol. 2014;261(3):579-88.

20. Hernandez-Diaz S, Smith CR, Shen A, Mittendorf R, Hauser WA, Yerby M, et al. Comparative safety of antiepileptic drugs during pregnancy. Neurology. 2012;78(21):1692-9.

21. Mawer G, Briggs M, Baker GA, Bromley R, Coyle H, Eatock J, et al. Pregnancy with epilepsy: obstetric and neonatal outcome of a controlled study. Seizure. 2010;19(2):112-9.

22. Meador KJ, Baker GA, Finnell RH, Kalayjian LA, Liporace JD, Loring DW, et al. In utero antiepileptic drug exposure: fetal death and malformations. Neurology. 2006;67(3):407-12.

23. Miskov S, Gjergja-Juraski R, Cvitanovic-Sojat L, Bakulic TI, Fucic A, Bosnjak-Pasic M, et al. Prospective surveillance of Croatian pregnant women on lamotrigine monotherapy-aspects of pre-pregnancy counseling and drug monitoring. Acta Clin Croat. 2009;48(3):271-81.

24. Cassina M, Dilaghi A, Di Gianantonio E, Cesari E, De Santis M, Mannaioni G, et al. Pregnancy outcome in women exposed to antiepileptic drugs: teratogenic role of maternal epilepsy and its pharmacologic treatment. Reprod Toxicol. 2013;39:50-7.

25. Dolk H, Jentink J, Loane M, Morris J, de Jong-van den Berg LT, EUROCAT Antiepileptic Drug Working Group. Does lamotrigine use in pregnancy increase orofacial cleft risk relative to other malformations? Neurology. 2008;71(10):714-22.

26. Werler MM, Ahrens KA, Bosco JL, Mitchell AA, Anderka MT, Gilboa SM, et al. Use of antiepileptic medications in pregnancy in relation to risks of birth defects. Ann Epidemiol. 2011;21(11):842-50.

27. Hunt S, Russell A, Smithson WH, Parsons L, Robertson I, Waddell R, et al. Topiramate in pregnancy: preliminary experience from the UK Epilepsy and Pregnancy Register. Neurology. 2008;71(4):272-6.

28. Ornoy A, Zvi N, Arnon J, Wajnberg R, Shechtman S, Diav-Citrin O. The outcome of pregnancy following topiramate treatment: a study on 52 pregnancies. Reprod Toxicol. 2008;25(3):388-9.

29. Margulis AV, Mitchell AA, Gilboa SM, Werler MM, Mittleman MA, Glynn RJ, et al. Use of topiramate in pregnancy and risk of oral clefts. Am J Obstet Gynecol. 2012;207(5):405.e1-e7.

30. Mawhinney E, Craig J, Morrow J, Russell A, Smithson WH, Parsons L, et al. Levetiracetam in pregnancy: results from the UK and Ireland epilepsy and pregnancy registers. Neurology. 2013;80(4):400-5. 
31. ten Berg K, Samren EB, van Oppen AC, Engelsman M, Lindhout D. Levetiracetam use and pregnancy outcome. Reprod Toxicol. 2005;20(1):175-78.

32. Fujii H, Goel A, Bernard N, Pistelli A, Yates LM, Stephens S, et al. Pregnancy outcomes following gabapentin use: results of a prospective comparative cohort study. Neurology. 2013;80(17):1565-70.

33. Guttuso T Jr, Robinson LK, Amankwah KS. Gabapentin use in hyperemesis gravidarum: a pilot study. Early Hum Dev. 2010;86(1):65-6.

34. Montouris G. Gabapentin exposure in human pregnancy: results from the Gabapentin Pregnancy Registry. Epilepsy Behav. 2003;4(3):310-7.

35. Morrow J, Russell A, Guthrie E, Parsons L, Robertson I, Waddell $\mathrm{R}$, et al. Malformation risks of antiepileptic drugs in pregnancy: a prospective study from the UK Epilepsy and Pregnancy Register. J Neurol Neurosurg Psychiatry. 2006;77(2):193-8.

36. Meischenguiser R, D'Giano CH, Ferraro SM. Oxcarbazepine in pregnancy: clinical experience in Argentina. Epilepsy Behav. 2004;5(2):163-7.

37. Kaaja E, Kaaja R, Hiilesmaa V. Major malformations in offspring of women with epilepsy. Neurology. 2003;60(4):575-9.

38. Viinikainen K, Heinonen S, Eriksson K, Kalviainen R. Community-based, prospective, controlled study of obstetric and neonatal outcome of 179 pregnancies in women with epilepsy. Epilepsia. 2006;47(1):186-92.
39. Artama M, Auvinen A, Raudaskoski T, Isojarvi I, Isojarvi J. Antiepileptic drug use of women with epilepsy and congenital malformations in offspring. Neurology. 2005;64(11):1874-78.

40. Hvas CL, Henriksen TB, Ostergaard JR, Dam M. Epilepsy and pregnancy: effect of antiepileptic drugs and lifestyle on birthweight. BJOG. 2000;107(7):896-902.

41. Samren EB, van Duijn CM, Christiaens GC, Hofman A, Lindhout D. Antiepileptic drug regimens and major congenital abnormalities in the offspring. Ann Neurol. 1999;46(5):739-46.

42. Holmes LB, Hernandez-Diaz S. Newer anticonvulsants: lamotrigine, topiramate and gabapentin. Birth Defects Res A Clin Mol Teratol. 2012;94(8):599-606.

43. Holmes LB, Baldwin EJ, Smith CR, Habecker E, Glassman L, Wong SL, et al. Increased frequency of isolated cleft palate in infants exposed to lamotrigine during pregnancy. Neurology. 2008;70(22 Pt 2):2152-8.

44. Dolk H, van den Berg Lde J, Loane M, Wang H, Morris J. Newer anticonvulsants: lamotrigine. Birth Defects Res A Clin Mol Teratol. 2012;94(11):959 (author reply 960).

45. Mines D, Tennis P, Curkendall SM, Li DK, Peterson C, Andrews $\mathrm{EB}$, et al. Topiramate use in pregnancy and the birth prevalence of oral clefts. Pharmacoepidemiol Drug Saf. 2014;23(10): 1017-25.

46. Vajda FJ, Graham J, Roten A, Lander CM, O'Brien TJ, Eadie M. Teratogenicity of the newer antiepileptic drugs-the Australian experience. J Clin Neurosci. 2012;19(1):57-9. 